\title{
Free vibration analysis of FGM plates based on Rayleigh-Ritz method
}

\author{
Jun Zhang', Tianyun $\mathrm{Li}^{2}$, Xiang $\mathrm{Zhu}^{3}$ \\ School of Naval Architecture and Ocean Engineering, Huazhong University of Science and Technology, \\ Wuhan, 430074, China \\ China Ship Development and Design Center, Wuhan, 430064, China \\ Collaborative Innovation Center for Advanced Ship and Deep-Sea Exploration, Shanghai, 200240, China \\ ${ }^{2}$ Corresponding author

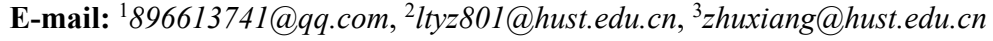

Received 12 November 2018; accepted 26 November 2018

DOI https://doi.org/10.21595/vp.2018.20368

Check for updates

Copyright (C) 2018 Jun Zhang, et al. This is an open access article distributed under the Creative Commons Attribution License, which permits unrestricted use, distribution, and reproduction in any medium, provided the original work is properly cited.

\begin{abstract}
Rayleigh-Ritz method was introduced to analyze the vibration characteristics of functionally graded material (FGM) rectangular plate with complex boundary conditions. The Improved Fourier series was chosen as the admissible function for its great property to be used universally in various boundary conditions. The virtual spring model was adopted to simulate the complex boundary conditions. The strain and kinetic energy of the structure and elastic potential energy of the border were easily calculated by using relevant formula. Finally, comparing the result with the references, the accuracy and convergence can be proved.
\end{abstract}

Keywords: FGM plate, Rayleigh-Ritz method, energy method, complex boundary conditions.

\section{Introduction}

In recent years, a rapid development in science and technology nowadays motivated researchers to researching new kinds of materials. Being widely used in automotive, ocean engineering, aerospace, electronic, and nuclear industries because of its outstanding advantage such as good adaptability to temperature gradients or easy to get desired strength and stiffness, functionally graded materials (FGMs) are attracting more attention day by day.

A large number of methods have been used to deal with the vibration problems of FGM plates. For numerical method is better at problems of structures with complex geometry, various methods were proposed mainly based on the finite element method or meshless methods [1]. J. Yang [2] developed a one-dimensional differential quadrature approximation and the Galerkin procedure to analyse the free vibration, and the Modal Superposition Method was used to analyze the transient response of the plate structure. Relevant numerical methods have been well developed.

As for analytical methods, T. Y. Ng [3] analyse the effects of FGM materials plate structures on the parametric resonance of based on the assumed mode method and Hamilton's principle. J. Woo [4] used an analytical solution to solve the problems of FGM plates for the nonlinear free vibration behaviour combining mixed Fourier series and von Karman theory. Sh. Hosseini-Hashemi [5] proposed an analytical solutions for vibration problems of FGM plates with elastic conditions. The analytical method was applied to the calculation of vibration problem of functionally gradient plates well, yet more research is needed as well.

In this paper, Rayleigh Ritz method was researched for solving the vibration problems of FGM plate. The improved Fourier series was chosen as the admissible function so that the traditional Rayleigh Ritz method can be used to calculate complex boundaries. Combining the first order shear deformation theory, the accuracy can be ensured. A numerical method was introduced to calculate the integration so that the efficiency of calculation is greatly improved. Numerical examples were given to prove the feasibility for solving the problem. Providing a reliable method for actual engineering problems. 


\section{Geometric models and properties of materials}

Set an FGM rectangular plate as an example. The sale is length $a$, width $b$ and total thickness $h$. The plate is made from a mixture of two kinds of materials, and the composition is described as follows. The formulations are deduced here with the assumption of the linear elastic material behavior and small displacements and strains. The material properties varied with plate thickness can be expressed as:

$X(z)=X_{m} V_{m}(z)+X_{c} V_{c}(z)$,

where $X$ denotes the effective material property such as density, Young's modulus etc. $X_{m}$ and $X_{c}$ is the properties of the two different materials. $V_{m}$ and $V_{c}$ are the volume fraction of the material according to the conditions of:

$V_{m}(z)+V_{c}(z)=1$

$V_{m}=\left(\frac{2 z+h}{2 h}\right)^{k},(k \geq 0)$.

In which $k$ is the volume fraction exponent. Taking Eqs. (3) into the Eqs. (1), the following equation can be obtained:

$X(z)=\left(X_{m}-X_{c}\right)\left(\frac{2 z+h}{2 h}\right)^{k}+X_{c}$

The relation between volume fraction and exponent is shown in Fig. 1 [6].

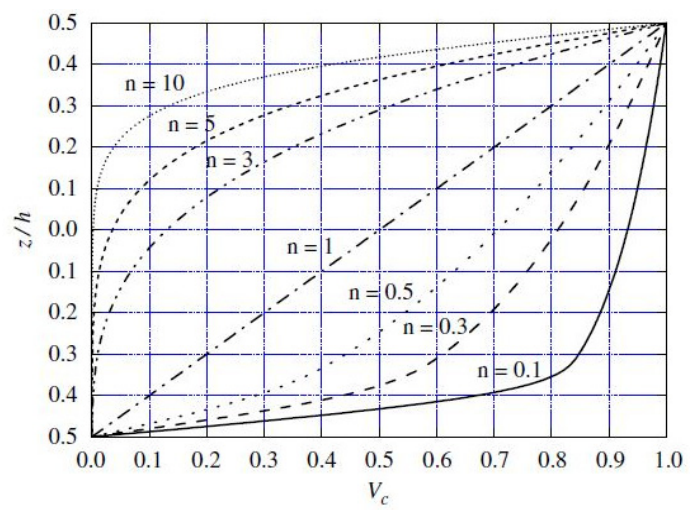

Fig. 1. Variation of the volume fraction through the thickness

\section{Theoretical formulations}

In the study, the Rayleigh Ritz method was introduced to solve the problem. Before derived the characteristic equation, a suitable admissible functions shall be given through which the specific expression of strain energy, kinetic energy and elastic potential energy can be obtained.

Considering the first order shear theory, suppose admissible functions of the rectangular FGM plates can be expressed as:

$w(x, y)=\sum_{m=1}^{M} \sum_{n=1}^{N} A_{m n} \varphi_{m}(x) \psi_{n}(y) e^{i \omega t}$,
$\beta_{x}(x, y)=\sum_{m=1}^{M} \sum_{n=1}^{N} B_{m n} \varphi_{m}(x) \psi_{n}(y) e^{i \omega t}$, 
$\beta_{y}(x, y)=\sum_{m=1}^{M} \sum_{n=1}^{N} C_{m n} \varphi_{m}(x) \psi_{n}(y) e^{i \omega t}$

In which $w(x, y)$ is the flexural displacement, $\beta_{x}(x, y)$ is the rotation angle of the normal line along the $x z$ plane, and $\beta_{y}(x, y)$ is the rotation angle of the normal line along the $y z$ plane, $A_{m n}$, $B_{m n}, C_{m n}$ are unknown coefficients, $\varphi_{m}(x), \psi_{n}(y)$ are functions related to $x$ and $y$ respectively, $e^{i \omega t}$ is harmonic time factor. The improved Fourier series is selected as the admissible function because it can satisfy arbitrary boundary conditions so that the virtual spring model can be introduced to simulate various complex boundary conditions, which can be expressed as [7]:

$\varphi_{m}(x)=\sin (m \pi x), \quad 0<m<5$,

$\varphi_{m}(x)=\cos [(m-5) \pi x], m \geq 5$,

$\psi_{n}(y)=\sin (n \pi x), 0<n<5$,

$\psi_{n}(y)=\cos [(n-5) \pi x], n \geq 5$,

where $m=1,2,3, \ldots, M ; n=1,2,3, \ldots, N$.

According to the first order shear theory, the strain energy can be expressed as:

$$
\begin{aligned}
V_{p} & =\frac{1}{2} D(z) \int_{0}^{a} \int_{0}^{b}\left\{\left(\frac{\partial \beta_{x}}{\partial x}+\frac{\partial \beta_{y}}{\partial y}\right)^{2}-2(1-\mu) \frac{\partial \beta_{x}}{\partial x} \frac{\partial \beta_{y}}{\partial y}+\frac{1}{2}(1-\mu)\left(\frac{\partial \beta_{x}}{\partial y}+\frac{\partial \beta_{y}}{\partial x}\right)^{2}\right\} d x d y \\
& +\frac{1}{2} k_{0} G h \int_{0}^{a} \int_{0}^{b}\left\{\left(\beta_{x}+\frac{\partial w}{\partial x}\right)^{2}+\left(\beta_{y}+\frac{\partial w}{\partial y}\right)^{2}\right\} d x d y .
\end{aligned}
$$

In which $D(z)=E(z) h^{3} / 12\left(1-\mu^{2}\right)$ represent the bending stiffness, $E(z)$ is the Young's modulus varies with the thick. $\mu$ is the Poisson's ratio, $k_{0}=6 / 5$ represent the shear coefficient, $G(z)=E(z) / 2(1+\mu)$ is the shear stiffness, $h$ is the thickness.

The kinetic energy of plate can be given as:

$T=\frac{1}{2} \rho(z) h \omega^{2} \int_{0}^{a} \int_{0}^{b}\left\{w^{2}+\frac{1}{12} h^{2}\left(\beta_{x}{ }^{2}+\beta_{y}{ }^{2}\right)\right\} d x d y$,

where $\rho(z)$ is the mass density and $\omega$ is the angular frequency.

The virtual spring model is used, and the effect of the boundary conditions on the vibration can be transformed into the influence of the elastic potential energy at the boundary to the stiffness matrix. Introducing displacement restrained springs and corner restrained springs with adjustable stiffness coefficient along the edges shown as Fig. 2.

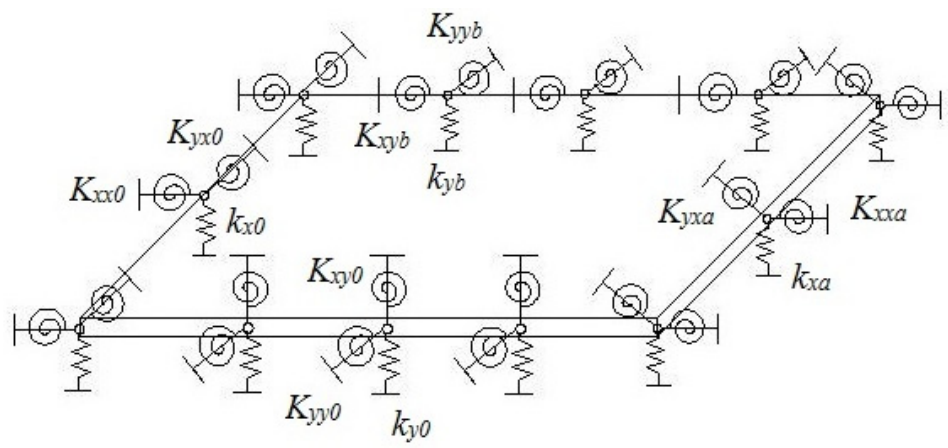

Fig. 2. Processing of boundary conditions

Supposing the displacement restrained springs constants and corner restrained springs 
constants are $k_{i j}\left(\mathrm{~N} / \mathrm{m}^{2}\right), K_{y i j}(\mathrm{~N} / \mathrm{rad})$ and $K_{x i j}(\mathrm{~N} / \mathrm{rad})$ respectively. It is easy to simulate various complex boundary conditions by adjusting the stiffness of the two kinds of springs. The simulation of classical boundary conditions is shown in Table 1 .

Table 1. Classical boundary conditions corresponding spring values

\begin{tabular}{|c|c|c|c|}
\hline & Clamped & Simply supported & Free \\
\hline$k\left(\mathrm{~N} / \mathrm{m}^{2}\right)$ & $\infty$ & $\infty$ & 0 \\
\hline$K(\mathrm{~N} / \mathrm{rad})$ & $\infty$ & 0 & 0 \\
\hline
\end{tabular}

Then the increased elastic energy at the boundary is:

$$
\begin{aligned}
V_{s} & =\frac{1}{2} \int_{0}^{b}\left\{\left[k_{x 0} w^{2}+K_{x x 0} \beta_{x}^{2}+K_{y x 0} \beta_{y}^{2}\right]_{x=0}+\left[k_{x a} w^{2}+K_{x x a} \beta_{x}^{2}+K_{y x a} \beta_{y}^{2}\right]_{x=a}\right\} d y \\
& +\frac{1}{2} \int_{0}^{a}\left\{\left[k_{y 0} w^{2}+K_{x y 0} \beta_{x}^{2}+K_{y y 0} \beta_{y}^{2}\right]_{y=0}+\left[k_{y b} w^{2}+K_{x y b} \beta_{x}^{2}+K_{y y b} \beta_{y}^{2}\right]_{y=b}\right\} d x .
\end{aligned}
$$

The energy functional of the whole structure can be expressed as:

$\Pi=V_{p}+V_{s}-T$.

Substituting Eqs. (7), (8), (9) into Eq. (10), and take the total energy for partial derivatives:

$\frac{\partial \prod}{\partial A_{m n}}=0, \frac{\partial \prod}{\partial B_{m n}}=0, \frac{\partial \prod}{\partial C_{m n}}=0$.

Then the vibration problem of the structure is transformed into the problem of solving eigenvalues, which can be expressed as follows:

$\left(\mathbf{K}-\omega^{2} \mathbf{M}\right) \mathbf{X}=\mathbf{0}$.

In which, $\mathbf{K}$ represent the stiffness matrix, $\mathbf{M}$ represent the mass matrix of the structure, $\mathbf{X}$ is the unknown coefficient vector.

\section{Numerical analysis}

When calculating, it takes a long time to calculate the integral. In order to improve the efficiency, numerical method is introduced. By dividing the plate structure into several small units, the calculation of integral is converted into calculating the summation.

Example 1. The values of truncated number $M, N$ in the admissible function have a great influence on the accuracy of the calculation. Square plate with free boundary condition (FFFF) was chosen as examples. The parameters of the model are as follows: the length is $a=1 \mathrm{~m}$, and the thickness is $h=0.1 \mathrm{~m}$. Table 2 compares the frequencies (for $h / a=0.1$ ) calculated by using different number of terms in the series. When $M=N=12$, the natural frequency tends to be a constant, which can be considered the method has converged.

Example 2. The length of the plate is $a=1 \mathrm{~m}$. When $k=0,0.5,1,4,10$, natural frequencies of the square FGM plates with different thickness to length ratios $(h / a=0.05$, and 0.2$)$ are given in Table 3 . The boundary condition are SSSS. The plates are made of aluminum ( $\mathrm{Al}, E=70 \mathrm{GPa}$, $\left.\rho=2702 \mathrm{~kg} / \mathrm{m}^{3}, \mu=0.3\right)$ and alumina $\left(\mathrm{Al}_{2} \mathrm{O}_{3}, E=380 \mathrm{GPa}, \rho=3800 \mathrm{~kg} / \mathrm{m}^{3}, \mu=0.3\right)$. By comparing the results with the references, the accuracy of the method can be proved.

Example 3. Changing the materials, Table 4. shows the results of the square plates with the length ratios of $h / a=0.1$ for the $\mathrm{Al} / \mathrm{ZrO}_{2}\left(\mathrm{ZrO}_{2}, E=200 \mathrm{GPa}, \rho=5700 \mathrm{~kg} / \mathrm{m}^{3}, \mu=0.3\right)$. Only the results for the first four modes are given. At the boundary conditions of SSSS and CFFF, 
comparing with the reference, the present method is accurate for solving the problems of FGM plates.

Table 2. Nature frequencies of rectangular plates $\left(\gamma=\omega a^{2} / h \sqrt{\rho_{m} / E_{m}}, \mathrm{Al} / \mathrm{Al}_{2} \mathrm{O}_{3},(h / a=0.1)\right)$

\begin{tabular}{|c|c|c|c|c|c|c|}
\hline Mode & $M=N=9$ & $M=N=10$ & $M=N=11$ & $M=N=12$ & $M=N=13$ & $M=N=14$ \\
\hline 1 & 2.96 & 2.95 & 2.95 & 2.94 & 2.94 & 2.94 \\
\hline 2 & 4.39 & 4.39 & 4.39 & 4.39 & 4.39 & 4.39 \\
\hline 3 & 5.41 & 5.41 & 5.41 & 5.41 & 5.41 & 5.41 \\
\hline 4 & 7.44 & 7.43 & 7.42 & 7.41 & 7.41 & 7.40 \\
\hline
\end{tabular}

Table 3. Comparison of natural frequencies of FGM plate $\left(\gamma=\omega h \sqrt{\rho_{m} / E_{m}}, \mathrm{SSSS}, \mathrm{Al} / \mathrm{Al}_{2} \mathrm{O}_{3}\right)$

\begin{tabular}{|c|c|c|c|c|c|c|}
\hline \multirow{2}{*}{$\delta=h / a$} & \multirow{2}{*}{ Reference } & \multicolumn{5}{|c|}{ Gradient index } \\
\cline { 3 - 7 } & & 0 & 0.5 & 1 & 4 & 10 \\
\hline \multirow{3}{*}{0.05} & Pre. & 0.01446 & 0.01228 & 0.01107 & 0.00960 & 0.00919 \\
\cline { 2 - 7 } & Ref. [5] & 0.01480 & 0.01281 & 0.01150 & 0.01013 & 0.00963 \\
\cline { 2 - 7 } & Ref. [6] & 0.01464 & 0.01241 & 0.01118 & 0.00970 & 0.00931 \\
\hline \multirow{3}{*}{0.2} & Pre. & 0.19861 & 0.16994 & 0.15367 & 0.13136 & 0.12430 \\
\cline { 2 - 7 } & Ref. [5] & 0.21120 & 0.18060 & 0.16500 & 0.13710 & 0.13040 \\
\cline { 2 - 7 } & Ref. [6] & 0.20550 & 0.17570 & 0.15870 & 0.13560 & 0.12840 \\
\hline
\end{tabular}

Table 4. Natural frequencies of FGM plate $\left(\gamma=\omega a^{2} / h \sqrt{\rho_{m} / E_{m}}, \mathrm{Al} / \mathrm{ZrO}_{2}, h / a=0.1\right)$

\begin{tabular}{|c|c|c|c|c|c|c|c|c|c|}
\hline \multicolumn{2}{|c|}{} & \multicolumn{4}{c|}{ SSSS } & \multicolumn{5}{c|}{ CFFF } \\
\hline Mode & - & 1 & 2 & 3 & 4 & 1 & 2 & 3 & 4 \\
\hline 0 & Pre. & 5.545 & 13.471 & 13.471 & 20.453 & 1.038 & 2.439 & 6.09 & 7.734 \\
\hline- & Ref. [6] & 5.676 & 13.537 & 13.537 & 20.633 & 1.03 & 2.391 & 6.005 & 7.636 \\
\hline 0.5 & Pre. & 4.997 & 12.149 & 12.149 & 18.465 & 0.934 & 2.197 & 5.486 & 6.965 \\
\hline- & Ref. [6] & 5.111 & 12.207 & 12.207 & 18.63 & 0.926 & 2.153 & 5.409 & 6.051 \\
\hline 1 & Pre. & 4.764 & 11.58 & 11.58 & 17.595 & 0.891 & 2.095 & 5.229 & 6.639 \\
\hline- & Ref. [6] & 4.871 & 11.633 & 11.633 & 17.748 & 0.883 & 2.052 & 5.155 & 5.76 \\
\hline 2 & Pre. & 4.592 & 11.152 & 11.152 & 16.923 & 0.86 & 2.02 & 5.042 & 6.402 \\
\hline- & Ref. [6] & 4.698 & 11.199 & 11.199 & 17.063 & 0.853 & 1.979 & 4.968 & 5.444 \\
\hline
\end{tabular}

\section{Conclusions}

The Rayleigh-Ritz method was introduced to analyze the free vibration characteristic. Combined with the first order shear theory, the accuracy can be ensured. Besides, virtual spring model was adopted to simulate the complex boundary conditions. Several examples were given, comparing the frequencies with the references, good convergence and accuracy can be proved. There is a unique advantage that the programming of the method is simple comparing with FEM when the parameter of the calculation model changed. What is important is that: the present method can be easily combined with other method when analyzing more complicated problems.

\section{References}

[1] Swaminathan K., Naveenkumar D. T., Zenkour A. M., Carrera E. Stress, vibration and buckling analyses of FGM plates - a state-of-the-art review. Composite Structures, Vol. 120, 2015, p. 10-31.

[2] Yang J., Shen Hui Shen Dynamic response of initially stressed functionally graded rectangular thin plates. Composite Structures, Vol. 54, Issue 4, 2001, p. 497-508.

[3] Ng T. Y., Lam K. Y., Liew K. M. Effects of FGM materials on the parametric resonance of plate structures. Computer Methods in Applied Mechanics and Engineering, Vol. 190, Issues 8-10, 2000, p. 953-962.

[4] Woo J., Meguid S. A., Ong L. S. Nonlinear free vibration behavior of functionally graded plates. Journal of Sound and Vibration, Vol. 289, Issue 3, 2006, p. 595-611. 
[5] Sh Hosseini Hashemi, Rokni Damavandi Taher H., Akhavan H., Omidi M. Free vibration of functionally graded rectangular plates using first-order shear deformation plate theory. Applied Mathematical Modelling, Vol. 34, Issue 5, 2010, p. 1276-1291.

[6] Zhao X., Lee Y. Y., Liew K. M. Free vibration analysis of functionally graded plates using the element-free KP-Ritz method. Journal of Sound and Vibration, Vol. 319, Issue 3, 2009, p. 918-939.

[7] Li W. L. Vibration analysis of rectangular plates with general elastic boundary supports. Journal of Sound and Vibration, Vol. 273, Issue 3, 2004, p. 619-635. 